

\section{Sumário}

A DISCRIMINAÇÃo RACIAL NO BRASIL E A ASCENSÃo DO POVO NEGRO: UM OLHAR A PARTIR DOS PRINCÍPIOS CONSTITUCIONAIS NA LUTA PELA CIDADANIA INCLUSIVA

Bruno Mello Correa de Barros e Rita Mara Albrecht

As MULHERES NO CONTEXTO DA SOCIEDADE DE CLASSES E SUA PARTICIPAÇÃO POLÍTICA NO PROCESSO LEGISLATIVO E EXECUTIVO DO BRASIL: RESTRIÇÕES E DESAFIOS

Rafael Bueno da Rosa Moreira e Marli Marlene Morais da Costa

FACTORES ASOCIADOS A LA VIOLENCIA DE GÉNERo EN PAREJAS ADOLESCENTES

Maria del Carmen Monreal Gimeno

Os IMPACTOS DAS TECNOLOGIAS DA INFORMAÇÃO NO FLUXO DE PESSOAS: VIOLAÇÕES DA LIBERDADE EM UM MUNDO SECURITIZADO

Elias Jacob de Menezes Neto, Jose Luis Bolzan de Morais e Victoria Layze Silva Fausto

El DELITO DE ENALTECIMIENTO TERRORISTA. ¿INSTRUMENTO DE LUCHA CONTRA EL PELIGROSO DISCURSO DEL ODIO TERRORISTA O MECANISMO REPRESOR DE REPUDIABLES MENSAJES DE RAPEROS, TWITTEROS Y TITIRITEROS?

Alfonso Galán Muñoz

As POLÍtiCAS PÚBLICAS E A PROMOÇão DA DIGNIDADE: UMA ABORDAGEM NORTEADA PELAS CApacidades (CAPABIlities APproach) propostas por Martha Nussbaum

Anna Paula Bagetti Zeifert e Janaína Machado Sturza

A INFLUÊNCIA DA MÍdia PARA O CONSUMO DE TABACO

Luís Renato Vedovato e Maria Carolina Gervásio Angelini

¿SON PARTE DEL BLOQUE DE CONSTITUCIONALIDAD LOS PRINCIPALES TRATADOS internacionales de derechos humanos de la ONU en Chile? Del texto positivo a la APLICACIÓN EN TRIBUNALES DE JUSTICIA Juan Pablo Díaz Fuenzalida

Potestad calificadora del Conservador de Bienes Raíces y Procedimiento Registral ....173 Sebastián Bozzo Hauri e Gonzalo Ruz Lartiga

DESARTICULAÇÃo INTERFEDERATIVA E CONCESSÃo DOS BENEFÍCIOS DE PRESTAÇÃo CONTINUADA (BPC) 
Aprendizagem Profissional e o poder público municipal: proposta de maior eficácia À POLÍTICA PÚBLICA

José Rodrigo Paprotzki Veloso

Direito A Conciliação Entre Trabalho E Família................................................229

Edilton Meireles de Oliveira Santos

Trabajo y diversidad funcional. La situación EN EL ORdenamiento JuRídico español 245 María Esther Carrizosa Prieto

Custos de TransaÇão como uma METAPolítica pública .........................................2276

João Luis Nogueira Matias e Ricardo José Brito Bastos Aguiar de Arruda

REVISITANDO O CONCEITO DE SERVIÇO PÚBLICO

Andre Luiz Dos Santos Nakamura

O Brasil FACE AOS NOVOS PADRÕES DE COMÉRCIO E INVESTIMENTO DOS ACORDOS INTERNACIONAIS

Michelle Sanchez Badin, Fabio Costa Morosini e David M. Trubek

Os contratos comerciais na Declaração dos Direitos de Liberdade Econômica (MP 881/19).

André Lipp Pinto Basto Lupi

O MARCO LEGAL DA INOVAÇÃO E O AUMENTO DA INTERAÇÃO ENTRE UNIVERSIDADE E EMPRESA: CONTRIBUIÇÕES PARA A CONSOLIDAÇÃO DO DIREITO FUNDAMENTAL AO DESENVOLVIMENTO ....352 Thiago Paluma e Eline Débora Teixeira

RELICITAÇÃo DAS CONCESSÕES DE GERAÇÃo DE ENERGIA ELÉTRICA E A REVERSÃo DE BENS....372 Patrícia Regina Pinheiro Sampaio e Sergio Guerra

FinANCIAMENTO TRANSGERACIONAL DA INFRAESTRUTURA VERDE FLORESTAL: O SISTEMA DE PAGAMENTO POR SERVIÇOS AMBIENTAIS COMO INSTRUMENTO DE GESTÃO DE RISCOS NA SOCIEDADE CONTEMPORÂNEA...................................................................................................390

Délton Winter de Carvalho e Kelly de Souza Barbosa

Mediação como Política Pública para Tratamento de Conflitos Consumeristas ..... 415 Fernanda Sartor Meinero e Fernando Pedro Meinero

A ParticipaÇão do AMICUS CURIAE EM DECISÕES JUdiCIAIS E SUA CONSEQUENTE CONTRIBUiÇÃo para efetivação de Políticas Públicas .429

Viviane Nobre Santana 
A ISONOMIA TRIBUTÁRIA COMO LIMITE À TRIBUTAÇÃo E À CONCESSÃO DE ISENÇÕES FISCAIS, E A INEFETIVIDADE DO CONTROLE JURISDICIONAL DESSAS ISENÇÕES..................................450

Paulo Alves da Silva Paiva e Alexandre Augusto Batista de Lima

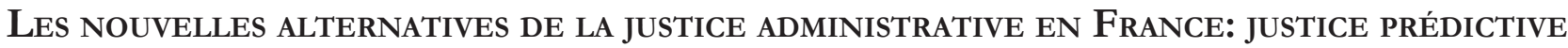
ET JUSTICE AMIABLE

Marie-Odile Diemer

O ESTADO DA LUXÚRIA: A PARÁBOLA DO BMW E A REAL DIMENSÃo DO DEBATE SOBRE A RESERVA DO POSSÍVEL: ESCASSEZ DE RECURSOS OU ORDENAÇÃo DE PRIORIDADES?................................484 Assis José Couto do Nascimento

O PODER CONSTITUINTE

José Levi Mello do Amaral Júnior

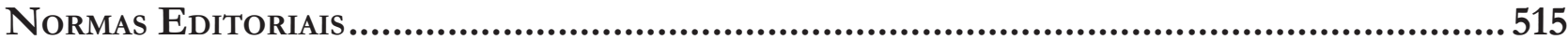

Envio dos trabalhos: 


\title{
Custos de transação como uma metapolítica pública*
}

\section{Transaction costs as a metapolicy}

\author{
João Luis Nogueira Matias** \\ Ricardo José Brito Bastos Aguiar de Arruda***
}

\section{Resumo}

Este artigo propõe a consideração original de custos de transação como uma metapolítica pública. O conceito vai além das relações entre firmas e mercado, afetando a integração das empresas, o bloqueio na formação de mercados, o funcionamento de sistemas econômicos, os custos governamentais, as barganhas entre facções políticas e sobre políticas entre eleitores e políticos. Explora as funções ou benefícios dos custos de transação, notadamente em situações de assimetria de informações e na escolha de arranjos institucionais. Discute a minimização dos custos de transação como meta, mas considerando a eficiência, percebendo que a formação de mercados pode reclamar a adição de funções e custos de transação, pois os agentes econômicos possuem racionalidade limitada. Passa a uma abordagem tópica em decisões legislativas, judiciais e corporativas, como em fusões e aquisições empresariais, mercados de capitais e questões de propriedade intelectual. A metodologia utilizada abrangeu a identificação do problema de eficiência na gestão pública, pesquisa do estado da arte acerca da manipulação do conceito de custo de transação, notadamente quanto à aplicação dessa ideia na governança pública, com a proposta de lançar a semente para o aprofundamento teórico e experimental da articulação de uma metapolítica pública centrada na ideia de custos de transação. O método é essencialmente descritivo-discursivo. $\mathrm{O}$ artigo recomenda a incorporação do conceito de custo de transação no processo de tomada de decisão pelo gestor público quanto às políticas públicas em geral, algumas vezes sendo esse um critério decisivo, outras não, mas sempre útil pelo impacto potencial na solução adotada.

Palavras-chave: Custos de transação. Metapolítica pública. Gestão Pública. Instituições. Eficiência.

** Doutor em Direito Comercial pela Universidade de São Paulo (2009), Doutor em Direito Público pela Universidade Federal de Pernambuco (2003), Mestre em Direito e Desenvolvimento pela Universidade Federal do Ceará (1999), Professor Associado, nível III, da Universidade Federal do Ceará e do Centro Universitário 7 de Setembro, Juiz Federal. Email: joaoluisnm@uol.com.br

*** Juiz Federal. Mestrando em Direito pela Universidade Federal do Ceará na área de concentração em Ordem Jurídica Constitucional; e Engenheiro Civil com especialização em Engenharia de Petróleo. Email: rjbbaa@yahoo.com

\section{Abstract}

This article proposes the original consideration of transaction cost as a metapolicy. This concept has expanded beyond firm-market relations, affecting corporate integration, blocking market formation, functioning of economic systems, government costs, bargaining among political factions and between voters and politicians. It explores the functions or benefits of transaction cost economics, highlighting their importance in situations of 
asymmetric information and in the choice of institutional arrangements. It discusses the minimization of transaction costs as a goal but in view of efficiency, realizing that the formation of markets may demand that functions and transaction costs be added, given that economic agents have a bounded rationality. A topical approach is taken in legislative, judicial and corporate decisions, such as mergers and acquisitions, capital markets and intellectual property issues. The methodology used throughout the present work was to identify the problem of efficiency in public management, by the research about the state of the art about the manipulation of the concept of transaction cost, especially with regard to the application of this idea in public governance, with the proposal to launch the seed for the theoretical and experimental deepening of the articulation of a metapolicy centered on the idea of transaction costs. The method was essentially descriptive-discursive. It is concluded by the recommendation of the use of transaction costs in the decision-making process by the public manager in relation to public policies in general, sometimes being a decisive criterion, others not, but always useful because of the potential impact on the solution adopted.

Keywords: Transaction cost economics. Metapolicies. Public management. Institutions. Efficiency.

\section{Introdução}

A importância dos custos envolvidos nas transações econômicas foi primeiramente percebida por Ronald Coase, quando procurou explicar a razão da existência da firma, bem como o porquê de seu tamanho. Com o tempo, essa ideia passou a ser empregada em áreas distintas da análise da firma, estando, hoje, presente nos mais diversos estudos de Economia e do Direito e Economia (Law and Economics). É impossível, por exemplo, elaborar uma teoria dos contratos_-vide os estudos de Oliver Hart e de Bengt Holmstrom¹-, identificar a melhor estratégia para pôr em prática uma determinada política pública, comparar instituições ${ }^{2} e$ prever os efeitos de uma nova legislação sem o domínio dos custos de transação, sejam reais ou virtuais.

Oliver E. Williamson ${ }^{3}$, debruçando-se sobre o estudo da governança das organizações, manipula o conceito de custos de transação. Elinor Ostrom ${ }^{4}$, do mesmo modo, ao desenvolver os seus trabalhos acerca da governança de sistemas econômicos complexos. Não apenas no campo da microeconomia, mas também na macroeconomia faz-se importante o domínio e o manejo dessa importante ferramenta. Joseph E. Stiglit$z^{5}$ chama a atenção para a resposta da Teoria Macroeconômica à virada em prol da microeconomia ocorrida nos anos setenta, resposta construída por uma nova macroeconomia baseada na microeconomia tradicional, mas reconhecendo as falhas de mercado. Sendo o custo de transação um conceito essencial ao estudo da microeconomia, essa nova abordagem da macroeconomia pós-keynesiana dele não pôde prescindir.

Pretende-se, com o presente artigo, a proposição da utilização do conceito de custos de transação como uma metapolítica pública, ou seja, demonstrar a importância do manuseio dessa ferramenta microeconômica na escolha, elaboração e acompanhamento das políticas públicas de modo geral. Os custos de transação

\footnotetext{
HART, O.; HOLMSTRÖM, B. Contract Theory. Stockholm: The Royal Swedish Academy of Sciences, 2016. Disponível em: $<$ https://www.nobelprize.org/nobel_prizes/economic-sciences/laureates/2016/advanced-economicsciences 2016.pdf>. Acesso em: 25 jun. 2017.

2 COLE, D. H. The Varieties of Comparative Institutional Analysis. Wisconsin Law Review, Madison, p. 383-409, 2013.

3 WILLIAMSON, O. E. Transaction Cost Economics: the natural progression. Prize Lecture. Berkeley: University of California, 2009. p. 455-476. Disponível em: < http://www.nobelprize.org/nobel_prizes/economic-sciences/laureates/2009/ williamson_lecture. pdf>. Acesso em: 25 jun. 2017.

4 OSTROM, E. Beyond Markets and States: Polycentric Governance of Complex Economic Systems. Prize Lecture. Workshop in Political Theory and Policy Analysis. Bloomington: Indiana University; and Tempe: Arizona State University, 2009. p. 408-444. Disponível em: <http://www.nobelprize.org/nobel_prizes/economic-sciences/laureates/2009/ostrom_lecture.pdf>. Acesso em: 25 jun. 2017.

5 STIGLITZ, J. E. Information and The Change in The Paradigm in Economics. Prize Lecture. New York: Columbia Business School, Columbia University, 2001. p. 472-540. Disponível em: <https://edisciplinas.usp.br/pluginfile.php/17485/mod_resource/content/1/CHY\%20-\%20Stiglitz\%20-\%20Aula\%205.pdf>. Acesso em: 25 jun. 2017.
} 
devem entrar na gramática do gestor público, ser-lhe familiar, compor o acervo ferramental para a tomada de decisões. Obviamente, não se está a propor que o critério econômico baseado no custo de transação seja, sempre, o fator determinante para a tomada de decisões quanto às variadas políticas públicas, mas que não deva ser ignorado. Qualquer que seja a opção que se faça, tem-se que estar consciente das consequências em termos de custos de transação.

O método é essencialmente descritivo-discursivo, sendo o artigo dividido em três capítulos. No primeiro, definem-se custos de transação, abordando a construção do conceito que contou com a contribuição de diversos teóricos, o que a tornou útil para a compreensão e solução de problemas que se verificam sempre que uma relação de troca se faz presente.

Segue-se, com o esclarecimento acerca das funções e benefícios dos custos de transação, afastando-se a ideia intuitiva de que, por serem custos, são necessariamente indesejáveis.

Ainda no primeiro capítulo, analisa-se, em postura crítica, a meta de minimização dos custos de transação. Pondera-se que a ideia de eficiência, eventualmente, pode ser aumentada com o incremento de custos, inclusive de transação, desde que tragam um resultado marginal superior ao custo marginal. Procura-se demonstrar que a própria formação de mercados pode depender da elevação dos custos de transação existentes, conclusão que se afigura contraintutiva, a princípio, pois o senso comum conduz à conclusão de que os custos devem ser sempre minimizados de modo a favorecer as trocas, mas que pode ser bem compreendida a partir da aceitação de que os agentes econômicos possuem uma racionalidade limitada (bounded rationality), o que justificaria a presença de facilitadores à transação os quais trazem consigo custos embutidos.

O segundo capítulo faz uma abordagem tópica, discutindo o tratamento de custos de transação nas decisões legislativas e judiciais, e destacando o papel fundamental dos advogados em importantes situações de assimetria de informações, como em fusões e aquisições de empresas, em mercados de capitais e em questões de propriedade intelectual.

Após todo esse escorço teórico, chega-se no terceiro capítulo ao ponto central do trabalho que consiste na proposição da consideração dos custos de transação como uma metapolítica pública, tomando por base um exemplo prático no contexto de uma política ambiental para o controle de emissão de gases por indústrias, discutindo as alternativas de criação de um mercado de carbono ou de exigência de redução eficiente e não uniforme de emissão para as plantas industriais.

Por fim, deixa-se claro que não se poder perseguir a redução dos custos de transação como uma metapolítica pública, um critério decisivo no desenho das políticas públicas, recomendando-se, antes, que o gestor público tenha consciência da existência dessa classe de custos e que o considere como um elemento importante em suas tomadas de decisão.

O tema abordado, pois, insere-se no estudo do direito associado à economia (Law and Economics), mas sob uma perspectiva que procura dar um passo além em relação ao que já foi desenvolvido a partir do trabalho pioneiro de Ronald Coase, como seja, na proposição de incorporação da ideia na atuação da Administração Pública, como uma metapolítica pública, de modo que a análise dessa classe de custos se faça presente na condução de todas as políticas públicas, assim como já ocorre, por exemplo, com a metapolítica pública de transparência.

\section{Custos de transação: conceito, funções e a meta de minimização como padrão de aplicação}

A utilização da noção de custos de transação como uma metapolítica pública enriquece a análise econômica e contábil das diversas políticas públicas postas em prática na gestão governamental, trazendo mais 
racionalidade às decisões administrativas ao evidenciar determinados custos que, de outro modo, poderiam passar desapercebidos, ao mesmo tempo em que ressalta a utilidade de funções agregadas às transações que, conquanto tragam consigo custos adicionais, permitem prevenir a emergência de problemas e adicionam um ganho marginal vantajoso.

Na busca pela demonstração da sua utilização na aplicação das políticas públicas, passa-se à análise do seu conceito, funções e padrão de aplicação.

\subsection{Conceito de custos de transação}

O trabalho pioneiro que evidenciou a existência de custos de transação foi desenvolvido por Ronald Coase em 1937. Trata-se do artigo The Nature of the Firm. Importava a Ronald Coase 6 fornecer um critério racional de escolha entre a produção de um bem ou serviço pela própria firma (adquirindo outras firmas ou contratando mais empregados) ou por meio de terceirização (adquirindo de outras firmas). Coase concluiu que, se o custo de contratar a obtenção de bens e serviços fosse demasiado alto, as firmas poderiam optar pela integração e regulação própria da atividade por meio da hierarquia da estrutura corporativa. A esses custos de contratação ele nomeou "custos de transação", ou seja, o custo de usar o sistema de preços (cost of using the price system).

Embora não tenha detalhado que custos seriam esses, Coase deixou claro que envolveriam custos de elaborar o contrato, de monitorar a performance desse contrato e de assegurar o cumprimento das obrigações contratuais. Segundo Driesen e Ghosh ${ }^{7}$, Coase, às vezes, pareceu sugerir que os custos de integração da firma também seriam custos de transação (custos para obter informações sobre trabalhadores a contratar, bem assim para monitorar o desempenho dos empregados, por exemplo — tais custos, para alguns, são denominados "custos de agência").

Essa definição de Coase foi por ele expandida quando escreveu The Problem of Social Cost ${ }^{8}$, de modo a tratar a possibilidade de barganha entre os proprietários de terra sobre questões envolvendo aborrecimentos derivados do uso do direito de propriedade (ruído, por exemplo). Custo de transação, portanto, também poder-se-ia referir ao custo dessa negociação. Coase cogitou, inclusive, do custo de se pagar alguém para que refreasse uma atividade nociva, como a poluição (the bribe contract).

Kenneth Arrows ${ }^{9}$ equiparou custos de transação com os custos que impedem ou bloqueiam a formação de mercados, ou ainda com os custos de funcionamento de um sistema econômico (certamente incluindo os custos governamentais para coagir o cumprimento dos contratos, bem assim para estabelecer os direitos de propriedade).

Douglas North ${ }^{10}$, do mesmo modo, implicitamente, incluiu os custos governamentais no conceito de custos de transação, notadamente quando os utilizou para comparar as economias capitalista (custos de mercado) e comunista (custos difíceis de medir, incluindo tempo para adquirir informações, em filas e outras perdas, tais como as decorrentes de monitoramento e coação imperfeitos) entre si.

Acadêmicos do direito têm utilizado um amplo conceito de custo de transação, abrangendo os custos do contrato privado e alguns dos custos para criar e operacionalizar soluções governamentais para problemas. Richard Posner, Neil Komesar e outros autores incluem o custo da tomada de decisão governamental

COASE, R. H. The Nature of The Firm. Economica, New Series, v. 4, n. 16, p. 386-405, 1937.

DRIESEN, M. D.; GHOSH, S. The functions of transaction costs: Rethinking Transaction Cost Minimization in a World of Friction. Arizona Law Review, Tucson, v. 47, p. 61-111, 2005.

8 COASE, R. H. The Problem of Social Cost. The Journal of Law \& Economics, Chicago, v. 3, 1960.

9 DRIESEN, M. D.; GHOSH, S. The functions of transaction costs: rethinking transaction cost minimization in a world of friction. Arizona Law Review, Tucson, v. 47, p. 61-111, 2005.

10 DRIESEN, M. D.; GHOSH, S. The functions of transaction costs: rethinking transaction cost minimization in a world of friction. Arizona Law Review, Tucson, v. 47, p. 61-111, 2005. 
como um custo de transação, segundo Driesen e Ghosh ${ }^{11}$. Komesar defende que os custos das alternativas institucionais devem ser levados em conta em uma análise comparativa (entre uma solução de mercado ou governamental, por exemplo). Para ele, devem ser separados os "custos de fricção" associados às alternativas, fazendo sentido tratá-los como custos de transação.

Conceitos mais amplos são utilizados por Robert Cooter e Avinash Dixit ${ }^{12}$, o primeiro abordando os custos de transação relativos à barganha entre facções políticas, enquanto Dixit se referindo aos custos envolvidos nas barganhas sobre políticas entre eleitores e políticos.

Uma larga definição de custos de transação permite uma justa comparação entre alternativas institucionais. Com efeito, atentar apenas para a redução dos custos de transação privados, ignorando eventual elevação nos custos de transação governamentais, resultará em opções equivocadas. Assim, os custos de transação devem abranger os custos administrativos associados com as ações governamentais, bem assim os custos privados associados com as transações de mercado, em ambos incluindo os custos de aquisição de informações necessárias para as decisões públicas e transações privadas.

Driesen e Ghosh ${ }^{13}$ definem custos de transação como sendo "os custos de lidar com as pessoas", incluindo os associados com a aquisição de informações tanto no âmbito público quanto privado. Onde não há relações pessoais, não há custos de transação. Pode haver custos, mas não custos de transação, porquanto impossível qualquer transação.

De maneira mais ampla ainda, defende-se que a existência de um corpo de leis que regule as atividades privadas favorece a redução dos custos de transação. Driesen e Ghosh ${ }^{14}$ referem esse tipo de assertiva como uma "afirmação de custo de transação fantasma" (phantom transaction cost claim), ou seja, trabalha com uma ideia do custo de transação que existiria se não houvesse essa regulação legal ou se o arranjo legal fosse distinto (custo de uma transação hipotética).

Assim, postula-se a aplicação do conceito mais largo de custos de transação.

\subsection{Funções ou benefícios dos custos de transação}

Os custos de transação existem e não podem ser desprezados. Importa definir que cumprem uma função útil em geral. Em situações de assimetria de informações, por exemplo, trabalham para equalizar o conhecimento para as partes que entabulam uma negociação. Com isso, propiciam uma maior eficiência da transação relativamente a quem detém menos informações, evitando a realização de contratos desequilibrados que, por sua vez, podem ocasionar grandes custos para economia, inclusive em decorrência da judicialização dos conflitos.

O conceito de custos de transação também é útil como um critério de comparação entre arranjos institucionais. Driesen e Ghosh ${ }^{15}$ informam que estudos legais, frequentemente, envolvem escolhas institucionais, sobre que instituições devem tomar decisões, o que reclama a análise de custos de transação. A decisão sobre permitir ao mercado privado ou deixar para a regulação estatal a definição das taxas ou preços dos serviços públicos é um exemplo de uma escolha institucional, frequentemente, sujeita à análise legal. Os custos de transação variam a depender da opção institucional feita.

11 DRIESEN, M. D.; GHOSH, S. The functions of transaction costs: rethinking transaction cost minimization in a world of friction. Arizona Law Review, Tucson, v. 47, p. 61-111, 2005.

12 DRIESEN, M. D.; GHOSH, S. The functions of transaction costs: rethinking transaction cost minimization in a world of friction. Arizona Law Review, Tucson, v. 47, p. 61-111, 2005.

13 DRIESEN, M. D.; GHOSH, S. The functions of transaction costs: rethinking transaction cost minimization in a world of friction. Ariqona Law Review, Tucson, v. 47, p. 61-111, 2005.

14 DRIESEN, M. D.; GHOSH, S. The functions of transaction costs: rethinking transaction cost minimization in a world of friction. Arizona Law Review, Tucson, v. 47, p. 61 - 111, 2005.

15 DRIESEN, M. D.; GHOSH, S. The functions of transaction costs: rethinking transaction cost minimization in a world of friction. Ariquna Law Review, Tucson, v. 47, p. 61-111, 2005. 
Driesen e Ghosh ${ }^{16}$, tratando da importância da informação e do custo de transação dedicado à sua obtenção, identificam três benefícios a ele aderentes: a) custos de transação ajudam a evitar ineficientes transações; b) possibilitam alcançar transações mais eficientes; e c) tornam as transações mais equitativas;

Em atenção a tais funções, esses autores defendem que recomendações para reduzir ou eliminar custos de transação devem, sempre, considerar o impacto da decisão sobre os benefícios a eles associados.

Os custos de transação abrangem o pagamento da aquisição e gerenciamento de informações. E adquirir informações é especialmente importante em situações de assimetria de conhecimentos entre as partes que desejam contratar. A informação, assim, seria tratada como uma commodity. A assimetria de informações provoca, pois, o nascimento de um mercado de informações. Caso não houvesse essa assimetria, inexistiria tal mercado.

Grossman e Stiglitz ${ }^{17}$, por exemplo, acreditam que se todas as pessoas tivessem iguais crenças e expectativas sobre o mercado de finanças, ele não existiria. A razão de sua ocorrência, portanto, deve-se ao fato de que, enquanto alguns estão dispostos a pagar um preço mais elevado por um ativo por reputá-lo mais valioso ou por esperar que ele se valorize, outros estão propensos a vender por acreditarem no oposto. Assim, ao contrário do que pensavam os economistas neoclássicos, informações corretas e completas não são um pré-requisito para um mercado perfeito, mas levariam ao fim do mercado. A despeito disso, é óbvio que melhores e mais completas informações tendem a tornar as transações mais eficientes, daí o custo de transação correspondente à sua aquisição. Informações perfeitas e completas implicariam transações plenamente eficientes e zero custo de transação, desde que tais transações fossem idealmente instantâneas.

Um vendedor, por exemplo, está disposto a pagar por informações acerca da solvabilidade de um comprador. Isso atrasa o negócio, torna-o mais caro, mas minimiza perdas e aborrecimentos. E como o custo do seguro também pode ser considerado de transação, mais informações podem adequar melhor o seu preço, eventualmente tornando o custo de transação global menor. Além disso, taxas de juros mais altas também são cobradas por conta da insegurança quanto ao pagamento. Em barateando essas taxas, mais negócios poderão ser realizados, o que implica ganho global.

Esse fenômeno foi muito bem identificado por Douglas North ${ }^{1819}$ ao tratar da história econômica da Inglaterra, em particular no contexto da Revolução Gloriosa, quando foi criado o Banco da Inglaterra e não mais se permitiu que o rei abusasse de seu poder para financiar as guerras extorquindo recursos da população inglesa. A prévia inspeção do bem a ser adquirido pelo comprador, do mesmo modo, evita-lhe aborrecimentos e dá-lhe segurança para pagar preços mais elevados, promovendo a realização de mais negócios.

Que fique claro que os custos de transação não se resumem àqueles informacionais. $\mathrm{Na}$ verdade, envolvem todos os vinculados à transação em si, tais como transporte, comunicação, coercibilidade, seguro e outros tantos. Às vezes, elevando-se um determinado custo de transação, reduz-se outro. Por exemplo, facilidades de comunicação podem reduzir os custos para a obtenção de informações ou relacionados a transporte. Mais informações podem implicar custo com seguro mais baixo.

Considerando-se as múltiplas e complexas funções dos custos de transação, importa verificar qual o padrão tradicional de sua abordagem.

\footnotetext{
DRIESEN, M. D.; GHOSH, S. The functions of transaction costs: rethinking transaction cost minimization in a world of friction. Arizona Law Review, Tucson, v. 47, p. 61-111, 2005.

17 DRIESEN, M. D.; GHOSH, S. The functions of transaction costs: rethinking transaction cost minimization in a world of friction. Arizona Law Review, Tucson, v. 47, p. 61-111, 2005.

18 NORTH, D. C. Institutions. Journal of Economic Perspectives, v. 5, n. 1, p. 97-112, 1991.

19 SALAMA, B. M. Sete Enigmas do Desenvolvimento em Douglas North. Latin American and Caribbean Law and Economics Association, 2011. Disponível em: <http://abdet.com.br/site/wp-content/uploads/2014/11/Sete-Enigmas-do-Desenvolvimento-emDouglass-North.pdf $>$. Acesso em: 25 jun. 2017.
} 


\subsection{Minimização dos custos de transação como meta}

O senso comum induz que se deve, sempre, trabalhar a redução de custos como um modo de evitar perdas e de maximizar os ganhos. Em um sentido amplo, nada há de errado com isso, pois a redução de custos, em regra, implica maior eficiência para a utilização dos recursos. A ideia de eficiência, por sua vez, está estreitamente vinculada à racionalidade, daí porque ser intencionada em qualquer área de atuação humana, seja no âmbito privado ou público (vide o caput do art. 37 da Constituição Federal na redação conferida pela EC 19/98). A busca na redução de custos, assim, é uma constante na atividade humana, seja otimizando processos, seja substituindo materiais caros por outros mais baratos e equivalentes, reduzindo o consumo de energia, reduzindo a mão de obra, cortando gastos desnecessários ou de qualquer outro modo.

Eficiência, todavia, é uma razão de duas variáveis: traduz-se pelo resultado alcançado dividido pelo resultado alcançável, considerados os recursos envolvidos. Pode-se, assim, não apenas elevar a eficiência pela redução dos recursos, reduzindo-se o resultado alcançável com a manutenção do alcançado, como também trabalhar para uma expansão no resultado alcançado sem alterar o alcançável, ou seja, sem a redução dos recursos envolvidos. Uma combinação das duas estratégias também é possível.

Aumentar os custos, portanto, pode ser uma estratégia para ampliar a eficiência, desde que os benefícios marginais superem os custos marginais. Em outras palavras, o incremento de benefício econômico pode superar o acréscimo de custo envolvido, elevando, com isso, a eficiência resultante.

Os custos de transação não funcionam de modo diverso. Muitas vezes, portanto, pode ser interessante elevá-los, desde que os benefícios promovidos sejam vantajosos, valham a pena. Evidentemente, elevar "custos mortos", ou seja, custos que não tragam benefícios, ou mesmo ineficientes, não seria uma estratégia inteligente.

A propósito, qualquer atividade humana envolve custos. Se o custo é zero, não há atividade, daí se dizer que a ausência de custos de transação somente é possível na inexistência de mercado. Custos de transação, portanto, são inerentes ao mercado. A despeito disso, Driesen e Ghosh ${ }^{20}$ advertem que a visão de que os custos de transação devem, sempre, ser reduzidos tem dado suporte a uma propensão pela adoção de livres mercados, tanto no âmbito teórico quanto na prática legal. Esse comportamento parte da equivocada e idealizada concepção de que os agentes econômicos detêm toda a informação necessária à tomada de decisões e que o fazem, sempre, racionalmente. Eric Posner ${ }^{21}$, por exemplo, comenta que os estudiosos de economia assumem que os indivíduos são racionais e que têm uma ilimitada capacidade cognitiva. Em razão disso, a teoria legal foi dominada, em largo período de tempo, por uma construção imaginária de um mundo de perfeita competição, completa informação e de nenhum custo de transação.

Oliver Williamson ${ }^{22}$, todavia, ganhador de prêmio Nobel dedicado ao estudo da "economia da governança" e da "economia das organizações", e que entende a governança como um conceito mais geral que utiliza os custos de transação como um mecanismo operacional, parte do princípio de que os agentes econômicos possuem uma "racionalidade limitada" (bounded rationality), o que leva a que todos os contratos complexos sejam incompletos, com aspectos da relação não regulados e obscuridades, demandando, com isso, o incremento de custos de transação para atenuar essa incompletude. Ronald Coase já insistia com a necessidade de se considerar o mundo real, e não ideal, sob a perspectiva da existência dos custos de transação ${ }^{23}$. Cass R.

\footnotetext{
20 DRIESEN, M. D.; GHOSH, S. The functions of transaction costs: rethinking transaction cost minimization in a world of friction. Arizona Law Review, Tucson, v. 47, p. 61 - 111, 2005.

21 DRIESEN, M. D.; GHOSH, S. The functions of transaction costs: rethinking transaction cost minimization in a world of friction. Arizona Law Review, Tucson, v. 47, p. 61- 11, 2005.

22 WILLIAMSON, O. E. Transaction cost economics: the natural progression. Prize Lecture. Berkeley: University of California, 2009. p. 455-476. Disponível em: <http://www.nobelprize.org/nobel_prizes/economic-sciences/laureates/2009/ williamson_lecture. pdf>. Acesso em: 25 jun. 2017.

23 DRIESEN, M. D.; GHOSH, S. the functions of transaction costs: rethinking transaction cost minimization in a world of friction. Ariqona Law Review, Tucson, v. 47, p. 61-111, 2005.
} 
Sunstein, Christine Jolls e Richard H. Thaler ${ }^{24}$ abordam a necessidade de que a análise econômica do Direito considere o real comportamento humano em suas escolhas.

Driesen e Ghosh ${ }^{25}$ constroem um exemplo interessante para explicar a relação entre mercado e custos de transação. Narram que houve um tempo em que os colonos assentados nos Apalaches do Kentucky tinham pouco contato com a economia de mercado. Por isso, não pagavam custos de transação. Os custos de transação eram zero e não havia mercado. Eles viviam isolados nas montanhas, cultivavam a terra e nada compravam em geral. Os autores assumem que o preço dos vestuários feitos nas cidades era barato o bastante para atrair o interesse dos fazendeiros se um mercador lhes apresentasse em suas fazendas. Mas, como nenhum mercador subia às montanhas, os fazendeiros ignoravam as possibilidades, e nenhum mercado de troca se estabelecia. Em algum momento, todavia, os fazendeiros começaram a comprar roupas feitas em outros lugares, pois os mercadores passaram a lhes apresentar as mercadorias, de modo que tomaram consciência dessa oportunidade. Os custos de transação teriam subido a tal ponto que permitiram o surgimento de um mercado. Nas condições anteriores, o real custo de transação era tão baixo que não possibilitava a existência de um mercado.

Driesen e Ghosh ${ }^{26}$ admitem que a ideia de os custos de transação serem tão baixos a impedir a formação de um mercado é contraintuitiva. Para a maioria dos economistas, o que ocorria no exemplo dado era que os custos de transação eram, ao contrário, tão elevados que não permitiam a formação de um mercado. Mas estariam eles a trabalhar com a ideia de custos de transação fantasma, e não de custos de transação real. Eles estariam a imaginar um diferente mercado que, se existisse, teria esses custos de transação elevados.

Driesen e Ghosh ${ }^{27}$ constroem uma situação hipotética particular que validaria essa ideia da maioria dos economistas. Assumem que subir às montanhas dos Apalaches era muito caro, de modo que ninguém poderia oferecer o vestuário se o preço devesse também incluir o custo de transação (fantasma). Mas, com a construção de uma estrada de ferro, o custo de transação (fantasma) caiu e viabilizou o mercado. Assumindo uma situação de perfeita informação e de comportamento racional de maximização do lucro, esse quadro estaria correto. Mas, em um mundo de racionalidade limitada (bounded rationality), o comerciante na cidade poderia atentar para a vizinhança na cidade, seus fornecedores, mas não prestaria atenção a todas as possibilidades em relação aos que poderiam levar as mercadorias até as montanhas (mercadores) e aos fazendeiros. Poderia ser que o custo fantasma, durante o período de subsistência, igualasse o real custo de transação inicial ao surgimento do mercado. Nesse caso, o custo de transação fantasma mantém-se constante e nada explica. O tempo de subsistência, assim, finda quando o real custo de transação cresce ao nível suficiente para criar um mercado.

Por definição, custo de transação pressupõe a existência de uma transação, seja ela real (custo de transação real), seja ela hipotética (custo de transação fantasma). Se não há mercado, não há de se falar em custo de transação real regular, mas apenas de custo de transação fantasma ou de custo de transação eventual. Seja como for, pode-se afirmar que um custo de transação muito baixo impede a formação de um mercado quando, para que de fato exista, seja necessário agregar atividades essenciais ao seu funcionamento. Tais atividades, por óbvio, implicam mais custos de transação, como no caso da introdução de um intermediário entre o fornecedor e o comprador. Mas são exatamente esses custos de transação que viabilizarão a formação do mercado. Em suma, não há mercado sem custos de transação. Custos de transação muito baixos,

${ }^{24}$ SUNSTEIN, C. R.; JOLLS, C.; THALER, R. H. A Behavioral Approach to Law and Economics. Chicago: The Law School - The University of Chicago, Program in Law and Economics Working Paper n. 55, 1998. Disponível em: <http://nrs.harvard.edu/urn3:HUL.InstRepos:12921734>. Acesso em: 30 abr. 2017.

25 DRIESEN, M. D.; GHOSH, S. The functions of transaction costs: rethinking transaction cost minimization in a world of friction. Arizona Law Review, Tucson, v. 47, p. 61-111, 2005.

26 DRIESEN, M. D.; GHOSH, S. The functions of transaction costs: rethinking transaction cost minimization in a world of friction. Arizona Law Review, Tucson, v. 47, p. 61-111, 2005.

27 DRIESEN, M. D.; GHOSH, S. The functions of transaction costs: rethinking transaction cost minimization in a world of friction. Ariquna Law Review, Tucson, v. 47, p. 61-111, 2005. 
do mesmo modo, podem impedir a formação do mercado, pelas razões acima. Ressalve-se que custos de transação elevados, como qualquer custo em geral, podem dificultar a formação do mercado.

\section{Custos de transação em decisões legislativas, judiciais e corporativas}

O anseio de redução dos custos de transação, como mencionam Driesen e Ghosh ${ }^{28}$, tem permeado a literatura do Law and Economics e influenciado, profundamente, as políticas públicas. Especificamente no que concerne às leis, tem-se focado, historicamente, mais no âmbito do direito privado relativamente ao conceito de custos de transação, muito embora o direito público também tenha trabalhado almejando a redução dos custos de transação, muitas vezes por meio da privatização. A privatização, diga-se, não de uma atividade econômica passando do Estado ao âmbito privado, mas de determinadas funções governamentais, ou seja, na forma de uma transferência para o âmbito privado de uma atividade pública dedicada à regulação da atividade privada, por exemplo, na política pública de redução de poluição, de desmatamento, de controle da pesca ou de bens de uso comum em geral.

Com efeito, pode ser mais economicamente vantajoso, e eficiente, criar e regular um mercado de carbono para se obter uma redução de emissão de gases poluentes do que, simplesmente, estabelecer limites de emissão de gases e fiscalizar o cumprimento dessa normatização. Essa seria uma justificativa racional para a utilização de mercados privados com a finalidade de satisfazer interesses de cariz público. Uma boa compreensão do conceito de custos de transação, portanto, pode auxiliar a decisão acerca da adequada solução institucional para enfrentar um determinado problema social, ora por meio de uma atuação direta do Estado, ora com o estímulo estatal a que a atividade privada, em perseguindo os seus próprios interesses, finde por atingir resultados socialmente mais vantajosos.

Está claro que as decisões legislativas impactam as instituições, públicas ou privadas, e o fazem, muitas vezes, alterando os custos de transação envolvidos. O legislador, portanto, deve considerar como os custos de transação serão afetados pela lei que propõe. A eliminação de um determinado custo de transação, certamente, implica a criação de outros que, no contexto geral da economia, podem ser ou não mais vantajosos, seja sob o prisma dos valores de custos de transação reciprocamente considerados, seja em razão dos benefícios suprimidos e gerados.

Ronald Coase ${ }^{29}$, em seu artigo "The Problem of Social Cost", afirmou que os conflitos entre proprietários de terra seriam melhor resolvidos por meio de entendimentos entre os proprietários (bargain), e não por meio de decisões judiciais amparadas no direito posto, na hipótese de essas barganhas serem isentas de custos de transação (essa ideia foi rotulada como "o teorema de Coase"). Com base nisso, Guido Calabresi e Douglas Melahmed ${ }^{30}$ propuseram que as cortes poderiam escolher entre a regra da propriedade (property rule) e a regra da responsabilidade (liability rule). Driesen e $\mathrm{Ghosh}^{31}$ explicam que a regra da propriedade usualmente protege o direito relativo à propriedade por meio de injunções, enquanto que a regra da responsabilidade o faz por meio de indenizações compensatórias. O debate em torno dessas duas opções legislativas se dá sob a consideração da solução mais eficiente em termos de custos de transação, inclusive no tratamento dos impedimentos a uma livre barganha das partes para corrigir decisões judiciais ineficientes.

\footnotetext{
28 DRIESEN, M. D.; GHOSH, S. The functions of transaction costs: rethinking transaction cost minimization in a world of friction. Arizona Law Review, Tucson, v. 47, p. 61- 11, 2005.

29 COASE, R. H. The Problem of Social Cost. The Journal of Law \& Economics, Chicago, v. 3, 1960.

30 DRIESEN, M. D.; GHOSH, S. The functions of transaction costs: rethinking transaction cost minimization in a world of friction. Arizona Law Review, Tucson, v. 47, p. 61-111, 2005.

31 DRIESEN, M. D.; GHOSH, S. The functions of transaction costs: rethinking transaction cost minimization in a world of friction. Ariqona Law Review, Tucson, v. 47, p. 61-111, 2005.
} 
Robert Cooter e Thomas Ulen ${ }^{32}$ dividiram o "Teorema de Coase" em duas partes: a) Teorema Positivo de Coase; e b) Teorema Normativo de Coase. O Teorema Positivo estabelece que, assumindo-se zero custo de transação, tem-se uma eficiente alocação de recursos por meio da barganha privada, não importando os direitos de propriedade inicialmente conferidos. Já o Teorema Normativo propõe que os legisladores deveriam laborar para remover os impedimentos à negociação privada, ou seja, reduzindo os custos de transação dessa negociação.

Há discussões em que se argumenta que as regras legais existentes, não as fantasmas, geram custos de transação excessivo, o que leva a sugestões de reformas legislativas, inclusive para privatizar a forma de obtenção do bem público.

Regras legais como a do art. 423 do Código Civil (quando houver no contrato de adesão cláusulas ambíguas ou contraditórias, dever-se-á adotar a interpretação mais favorável ao aderente) claramente elevam o custo de transação, pois induzem a um maior cuidado na redação do contrato, o que pode exigir a contratação de um advogado experiente para orientar na confecção do instrumento. Esse custo de transação gerado, todavia, cumpre a função de encorajar transações eficientes e equilibradas.

O juiz, muitas vezes, se depara com situações em que alternativas de resolução do conflito, todas elas igualmente defensáveis em termos jurídicos, se apresentam. A eficiência econômica ou, em termos mais estreitos, a minimização do custo de transação pode ser a chave para a seleção da decisão mais recomendável. Não se quer dizer com isso que o código de decisão "lícito-ilícito" deva ser substituído pelo código de decisão "ter-não ter" no âmbito do sistema jurídico, na linguagem de sistemas trabalhada por Luhmann e Marcelo Neves ${ }^{33}$. O critério econômico entraria no jurídico como informação devidamente filtrada por esse último sistema segundo os seus próprios critérios.

Um contrato que não fixa o preço do bem, por exemplo, poderia ser judicialmente complementado pela regra "default" segundo a qual se deve adotar um preço "razoável". Como alternativa, poder-se-ia tratar esse contrato como juridicamente não exigível. A definição da melhor solução pode passar pela comparação relativa dos custos de transação.

Em questões envolvendo direitos autorais, o custo de transação pode desempenhar um importante papel para a decisão judicial. A Lei 9.610/98 dispõe que a reprodução de uma obra legalmente protegida, ainda que parcial, depende da prévia e expressa autorização do autor, sendo, todavia, autorizada a reprodução, em apenas um exemplar, de pequenos trechos para uso privado do copista, desde que por ele realizada e sem o intuito de lucro. Por trás dessa autorização legislativa ao uso de obra legalmente protegida por outrem sem a necessidade de autorização do autor, por certo, está o conceito de custo de transação associado ao que seria um justo uso da obra.

Driesen e Ghosh ${ }^{34}$ informam que essa questão foi tratada nos Estados Unidos pelo "Copyright Act of 1976", provendo quatro fatores para determinar a justiça ou lealdade (fairness) do uso não autorizado: a) a natureza do uso; b) a natureza da obra; c) o quantum da violação da obra; e d) o efeito da infração no mercado potencial. Wendy Gordon ${ }^{35}$, por outro lado, argumentou que a existência de uma falha do mercado poderia justificar a lealdade ou justiça do uso. Isso ocorreria, por exemplo, quando o custo de transação para a obtenção da autorização excedesse o valor do trabalho obtido pelo usuário. Nesse caso, a se impor a necessidade de autorização, o usuário optaria pelo não uso, o que configuraria a ausência de mercado. Essa situação não se encontra expressamente albergada pela Lei dos Direitos Autorais brasileira. Abre-se a possibilidade, portanto, de a jurisprudência sanar essa ausência utilizando-se do conceito de custo de transação.

32 DRIESEN, M. D.; GHOSH, S. The functions of transaction costs: rethinking transaction cost minimization in a world of friction. Arizona Law Review, Tucson, v. 47, p. 61-111, 2005.

33 NEVES, M. A Constitucionalização Simbólica. 3. ed. São Paulo: Martins Fontes, 2011.

34 DRIESEN, M. D.; GHOSH, S. The functions of transaction costs: rethinking transaction cost minimization in a world of friction. Arizona Law Review, Tucson, v. 47, p. 61-111, 2005.

35 DRIESEN, M. D.; GHOSH, S. The functions of transaction costs: rethinking transaction cost minimization in a world of friction. Arizona Law Review, Tucson, v. 47, p. 61-111, 2005. 
Situações há, todavia, alertam Driesen e Ghosh ${ }^{36}$, em que não há falha de mercado, os custos de transação para a obtenção da licença são razoáveis, entretanto, não convém exigi-la por outras razões, tais como os direitos de ordem distinta dos usuários, tão importantes quanto os direitos autorais. Nas paródias, por exemplo, em razão de sua própria essência de se apoiar em trabalho já existente, bem assim no direito de comentar, particularidade do direito de livre expressão. A doutrina do justo uso (fair use) pode depender ou não dos custos de transação envolvidos e, portanto, da eventual falha de mercado. Em termos de custos de transação, todavia, quanto mais baixos forem eles em relação à obtenção de licença, mais estreitos serão os limites para a consideração de um uso justo que a dispense, tal como ocorre em ambientes digitais.

Outra hipótese envolve o conceito de custo de transação fantasma. Driesen e Ghosh ${ }^{37}$ exemplificam com a hipótese de um professor pagando para usar uma porção de um artigo em classe (transação fantasma). Se o custo associado a essa transação, custo para encontrar o titular do direito autoral e negociar a licença, for excessivo, estaria justificado o uso independente de autorização. Assim, um justo uso evitaria o pagamento de um custo de transação fantasma.

No caso American Geophysical v. Texaco ${ }^{38}$, por outro lado, decidiu-se que a cópia não autorizada de artigos científicos para propósitos de pesquisa não era um justo uso porque existia um ativo mercado estabelecido de licenças para fotocópias de artigos. Em outro caso nos Estados Unidos (Wide Church of God v. Philadelphia Church of God) ${ }^{39}$, decidiu-se que, desde que a necessidade de minimização do custo de transação não justificava a exclusão de licença, não estaria justificado o uso sem a licença devida.

Driesen e Ghosh ${ }^{40}$ relatam que a Suprema Corte americana tem, repetidamente, reconhecido que, com alguma frequência, precisa-se de mais custos de transação (mais processo) de modo a reduzir o risco de erro. Saber se um processo adicional é devido depende de uma avaliação relativa dos direitos em jogo, da estimativa do custo adicional, bem assim do esperado valor marginal das informações (benefícios) que serão incorporadas ao processo (Court's balancing test). A depender da gravidade com que o direito será afetado pela decisão, fará sentido agravar o custo do processo em busca de mais informações, ou seja, aceita-se um incremento no custo de transação que reduza as possibilidades de um dano causado por erro. Esse custo de transação pode tanto ser arcado pelas partes como pelo Estado, por óbvio.

A dignidade no devido processo legal também pode justificar custos de transação mais elevados. Driesen e Ghosh ${ }^{41}$ relatam que Jerry Mashaw teceu críticas à ênfase da Suprema Corte americana em evitar erros como o único valor a ser atendido pelo devido processo. Segundo Mashaw, há de se atentar, também, para as demandas por moralidade democrática, ou seja, para o fato de a participação da parte na construção de uma decisão que the afete aportar legitimidade ao processo. Isso pode justificar, por exemplo, a oportunidade de ouvir as razões da parte em audiência.

É muito comum a contratação de advogados quando se pretende realizar determinadas avenças, sendo isso verdade tanto em atividades pouco como muito reguladas. $\mathrm{O}$ aconselhamento de um advogado é conveniente para ambas as partes, pois pode auxiliar a clareza quanto ao estabelecimento dos direitos e obrigações, conduzir os interessados à percepção de desequilíbrios nos termos da avença, evitar desconformidades com

\footnotetext{
36 DRIESEN, M. D.; GHOSH, S. The functions of transaction costs: rethinking transaction cost minimization in a world of friction. Arizona Law Review, Tucson, v. 47, p. 61-111, 2005.

37 DRIESEN, M. D.; GHOSH, S. The functions of transaction costs: rethinking transaction cost minimization in a world of friction. Arizona Law Review, Tucson, v. 47, p. 61-111, 2005.

38 DRIESEN, M. D.; GHOSH, S. The functions of transaction costs: rethinking transaction cost minimization in a world of friction. Arizona Law Review, Tucson, v. 47, p. 61-111, 2005.

39 DRIESEN, M. D.; GHOSH, S. The functions of transaction costs: rethinking transaction cost minimization in a world of friction. Arizona Law Review, Tucson, v. 47, p. 61-111, 2005.

40 DRIESEN, M. D.; GHOSH, S. The functions of transaction costs: rethinking transaction cost minimization in a world of friction. Arizona Law Review, Tucson, v. 47, p. 61-111, 2005.

41 DRIESEN, M. D.; GHOSH, S. The functions of transaction costs: rethinking transaction cost minimization in a world of friction. Ariqua Law Review, Tucson, v. 47, p. 61-111, 2005.
} 
o ordenamento jurídico e, em suma, minimizar potenciais conflitos em relação à execução do contrato. Advogados, todavia, custam caro, integrando uma importante parcela dos custos da transação. Pode-se eliminar esse custo, por óbvio, mas essa opção, em regra, não é vantajosa em razão da elevada probabilidade de aparecerem outros custos de transação bem mais significativos, ou mesmo de se frustrar o resultado pretendido.

O conselho de um advogado se presta, também, para reduzir as assimetrias de informação, notadamente em áreas de grande complexidade jurídica, como em fusões e aquisições de empresas, mercado de capitais, além da aquisição de direitos relativos à propriedade intelectual, inclusive em áreas de conhecimento cuja exploração envolve um risco elevado (tecnologia da informação, por exemplo). O bem que o advogado vende é a informação, bem esse que tem um vital valor funcional. Esse é um claro exemplo de um custo de transação necessário. Minimizá-lo pode ser positivo, desde que os benefícios não sejam reduzidos em demasia ao ponto de tornar esta uma opção desvantajosa. Elevá-lo pode ser uma boa prática, a depender do ganho associado. Com efeito, os advogados podem adicionar valor à transação comercial.

\section{Custos de transação como metapolítica pública}

Nos Estados Unidos, a decisão acerca da opção de realizar políticas públicas diretamente pelo poder público ou terceirizando para o mercado tem levado em conta o conceito de custos de transação. Muitas vezes, todavia, concentra-se a crítica nos custos da burocracia envolvida na solução pública, mas despreza-se o custo da transação privada (ou mesmo pública) envolvido na alternativa. Isso ocorre, por exemplo, quando se pensa em substituir um serviço social público por uma alternativa, privada ou pública. Outras vezes, o contrário ocorre, como no caso do custo de transação privado no mercado de carbono que se busca reduzir, mas sem observar os custos de transação públicos envolvidos no monitoramento do mercado privado.

Por outro lado, há de se alinhar o interesse público de se atingir a meta desejada em relação a determinada política pública ao interesse privado de lucro, o que pode ser feito com o manejo adequado dos custos de transação, tanto públicos quanto privados. No caso da privatização dos sistemas prisionais, por exemplo, enquanto ao poder público interessa a consideração aos direitos fundamentais do preso e a sua reabilitação, o ente privado tende a centrar os esforços em uma eficiente detenção do preso (reclusão com segurança ao menor custo).

Driesen e Ghosh ${ }^{42}$ alertam que, em relação à comparação entre opções pública ou privada para a resolução de problemas, ou mesmo entre duas públicas, devem-se considerar os tipos de informações que as entidades eleitas necessitarão para realizar boas decisões funcionais, sendo este um primeiro passo para a construção da decisão. Assim, por exemplo, determinar o grau de detalhe da lei e o quanto se deve deixar para regulamentação administrativa depende da consideração das informações necessárias e dos custos de transação envolvidos em sua obtenção. O legislador tende a fazer uma apreciação da realidade com base em informações mais gerais que permitam enxergar os contornos da questão em decorrência do grau de fricção existente no processo legislativo e das limitações para a obtenção de dados detalhados. Deixa-se para a regulação administrativa, portanto, o detalhamento para a efetivação das decisões legislativas.

Seja como for, alertam os autores que a simples comparação entre os custos de transação alternativos não determina a melhor solução, se pública ou privada, para o problema. Isso porque outros fatores devem ser avaliados, tais como impedimentos para uma eficiente transação, ou mesmo se os eficientes mecanismos de mercado servem aos anseios da sociedade no contexto examinado.

Driesen e Ghosh ${ }^{43}$ mencionam que, no século XIX, havia um grande custo de transação no processo de

42 DRIESEN, M. D.; GHOSH, S. The functions of transaction costs: rethinking transaction cost minimization in a world of friction. Arizona Law Review, Tucson, v. 47, p. 61-111, 2005.

43 DRIESEN, M. D.; GHOSH, S. The functions of transaction costs: rethinking transaction cost minimization in a world of fric- 
indenização do trabalhador por acidentes de trabalho, porquanto demandava errática e cara litigância. Em tornando mais certa a responsabilidade do empregador independente de culpa, a adoção de um sistema de compensação no século XX eliminou custos de transação associados. Por outro lado, há quem advogue uma reforma em programas de bem estar social, defendendo que reduzir os custos de transação que dificultam a contratação de empregados (transporte, creches para crianças, informação sobre vagas de emprego) seria mais interessante economicamente do que simplesmente distribuir renda entre os pobres.

Essa discussão também se faz muito presente no Brasil quando se critica o Bolsa Família, conquanto os estudos realizados o qualifiquem como um programa bem-sucedido. Há sérias dúvidas sobre o uso do conceito de custos de transação para essa discussão, porquanto seria de difícil aferição a comparação relativa entre as alternativas. Talvez se deva trabalhar em ambas as frentes, promover a empregabilidade, mas sem uma política de precarização da relação de emprego, bem assim distribuindo renda, até porque essa política é bem recomendada pelas experiências Keynesianas.

Muito interessante é a utilização da ideia de custo de transação em questões ambientais, em particular no que concerne à adoção de um mercado de créditos de redução de emissão de gases (emissions trading). Driesen e Ghosh ${ }^{44}$ constroem o seguinte exemplo.

Suponha que o órgão estatal regulador queira obter a redução de 100 toneladas de gases poluentes de duas plantas industriais. Assim, cada uma deveria reduzir as suas emissões em 50 toneladas. Ocorre que a indústria A tem um custo marginal de controle de $\$ 10.000,00 /$ tonelada, enquanto que, para a indústria $\mathrm{B}$, esse custo marginal é de apenas $\$ 1.000,00 /$ tonelada. Assim, enquanto a indústria A teria um custo de $\$ 500.000$ para atender ao órgão regulador, a indústria B somente seria impactada em $\$ 50.000,00$. O custo total seria, portanto, \$550.000,00 para atingir a redução de poluentes desejada. Um comércio de emissões, todavia, poderia minimizar esse custo, permitindo que toda a redução seja arcada pela indústria B que, por sua vez, poderia vender o crédito de redução à indústria $\mathrm{A}$, não apenas ressarcindo-se do custo correspondente, mas tendo um lucro com essa venda. A empresa B, por sua vez, também sairia lucrando, pois poderia comprar esse crédito por um valor bem mais atraente do que o custo da própria redução. E o órgão regulador atingiria o objetivo da redução de 100 toneladas nas duas plantas.

Uma alternativa equitativa a esse mercado de crédito de redução de gases poderia ser desenhada a partir de uma exigência de redução eficiente e não uniforme de emissão para as plantas, mas o tempo e o custo de coletar as informações necessárias, bem como de controlar as emissões de cada planta, poderiam ser proibitivos. Uma análise em termos de custos de transação, portanto, pode ser uma interessante metapolítica pública para tratar a política pública de redução de poluentes. Existe uma assimetria de informações entre o órgão regulador e as indústrias relativamente aos custos envolvidos. Se não houvesse custos de transação para a obtenção dessas informações pelo órgão regulador, a alternativa proposta seria viável. Isso não ocorre, todavia. Imagine-se uma situação como essa envolvendo um polo industrial com centenas de plantas. Essa metapolítica pública, portanto, deve ser trabalhada de modo a formatar um mercado eficiente de créditos de carbono, por exemplo, de sorte a evitar fraudes, bem assim a reduzir os custos de transação do próprio mercado, tornando-o mais vantajoso do que a aflição de multas.

A necessidade de uma prévia aprovação por um órgão regulador para cada transação pode-se fazer necessária para evitar que sejam viciadas, não contribuindo adequadamente, com isso, para o resultado almejado. Com efeito, a qualidade do crédito não importa para o comprador, mas apenas para o regulador, salvo reflexamente pela qualidade do meio ambiente que a todos afeta. Deve, portanto, o poder público estabelecer um custo de transação adequado relativamente à aquisição de informação necessária a identificar a qualidade do crédito. A exigência de pública aprovação da aquisição de crédito também pode fazer sentido em

tion. Ariqona Law Review, Tucson, v. 47, p. 61-111, 2005.

44 DRIESEN, M. D.; GHOSH, S. The functions of transaction costs: rethinking transaction cost minimization in a world of friction. Arizona Law Review, Tucson, v. 47, p. 61-111, 2005. 
razão das diferenças geográficas em que localizadas as indústrias e as consequências ambientais distintas das poluições que produzem. Uma alternativa poderia ser restringir o mercado por regiões, mas, eventualmente, pode ser mais interessante para as partes reguladas arcar com custos de transação mais elevados do que um mercado de créditos mais restrito, pois este pode acabar por elevar os custos de transação ou tornar difícil a obtenção de crédito para um projeto desejado. Nada impede que se busque a redução dos custos de transação de modo responsável, mantendo-se ou incrementando-se a quantidade e qualidade das informações.

A depender do dano ambiental que se deseje combater, a geografia pode não ser o fator determinante, como pode ser o caso da chuva ácida e da camada de ozônio, situações em que a causa do dano pode estar geograficamente bem distante do local em que o efeito se revela.

Seja como for, qualquer que seja a política pública adotada com o intuito de reduzir a poluição ambiental (ou outra política pública, que o seja), somente fará sentido adotá-la se ela proporcionar um resultado superior àquele que seria obtido sem a sua adoção, ou seja, ausente a transação. A avaliação dos custos e benefícios da transação segue-se a esse primeiro filtro. Hão de se considerar, por óbvio, as consequências da não adoção da transação em termos de possível elevação na degradação ambiental. Manter o nível de risco ambiental pode ser justificável se a alternativa de uma não transação for o agravamento.

Custos de transação mais elevados, em uma determinada política pública, podem ser justificados por proporcionarem uma participação mais equitativa entre aqueles que devam ser considerados para a solução do problema para o qual lhes podem ser imputadas responsabilidades. Servem, portanto, para uma proporcional distribuição de responsabilidades. Isso pode ser muito claramente percebido em situações em que se busque a despoluição de um ambiente, algo como o princípio poluidor-pagador. Para tanto, é possível que o governo venha a ter necessidade de agregar informações para uma justa repartição de responsabilidades, elevando, com isso, os custos de transação da medida ou da política pública. O custo de transação, assim, financiaria uma escolha economicamente razoável, justa e efetiva para a solução do problema.

\section{Considerações finais}

O conceito de custo de transação não é uniforme entre os economistas ou profissionais do direito que adotam a abordagem do Direito e Economia. Evoluiu de uma primeira concepção que apenas percebia os custos de transação fora da firma, ou seja, no mercado de preços, talvez somente vislumbrando a existência de custos na estrutura hierárquica das corporações sem expressamente nominá-los como custos de transação ${ }^{45}$. Ampliou-se com a consideração dos custos de barganha entre agentes privados na solução de problemas derivados da propriedade de terras ${ }^{46}$. Kenneth Arrows voltou o olhar para os custos de funcionamento dos sistemas econômicos, aí incluindo os custos governamentais ${ }^{47}$. Foi tratado por Douglas North como um parâmetro para comparar modelos estatais de economia ${ }^{48}$. Utilizado por Richard Posner para o estudo do custo da tomada de decisão governamental, bem como por Neil Komesar para a análise comparativa de alternativas institucionais ou de escolha entre soluções de mercado ou governamental para alcançar metas públicas, com um olhar direcionado aos custos de transação enquanto custos friccionais ${ }^{49}$. Robert Cooter fez uma abordagem dos custos envolvidos nas barganhas entre facções políticas, enquanto Avinash Dixit

45 COASE, R. H. The nature of the firm. Economica, New Series, v. 4, n. 16, p. 386-405, 1937.

46 COASE, R. H. The problem of social cost. The Journal of Law \& Economics, Chicago, v. 3, 1960.

$47{ }^{49}$ DRIESEN, M. D.; GHOSH, S. The functions of transaction costs: rethinking transaction cost minimization in a world of friction. Arizona Law Review, Tucson, v. 47, p. 61-111, 2005.

48 DRIESEN, M. D.; GHOSH, S. The functions of transaction costs: rethinking transaction cost minimization in a world of friction. Arizona Law Review, Tucson, v. 47, p. 61-111, 2005.

49 DRIESEN, M. D.; GHOSH, S. The functions of transaction costs: rethinking transaction cost minimization in a world of friction. Arizona Law Review, Tucson, v. 47, p. 61-111, 2005. 
manejou o conceito para tratar de políticas entre eleitores e políticos ${ }^{50}$. Em todo caso, pode-se tomá-lo como abrangendo todo custo decorrente da transação em si ${ }^{51}$, seja informacional, de seguro, transporte, comunicação, de elaboração de contrato, registro, judicial, até mesmo o tempo, que pode ter um valor econômico.

Para além de sua importância fundamental nos estudos de micro e macroeconomia, em relação à compreensão sobre a existência e funcionamento da firma, no estudo dos contratos, da governança das organizações ou de sistemas econômicos complexos, incluindo a superação da tragédia dos comuns, percebe-se a necessidade imperiosa de incorporação do conceito de custo de transação no processo de tomada de decisão do gestor público no que concerne às políticas públicas em geral. Não se ignora que a preocupação sobre custos já esteja presente na teoria e prática envolvendo as escolhas de atuações, públicas ou privadas, que objetivam um determinado interesse público. O que se propõe, todavia, é uma particular abordagem nesse processo que leve em consideração, especificamente, os custos de transação, percebendo-os em sua peculiaridade e aproveitando toda a teorização já existente sobre esse conceito. Muitas vezes, uma análise comparativa dos custos de transação envolvidos nas alternativas de políticas públicas formuladas atuará como critério decisivo. Outras tantas, não, mas o gestor deve estar consciente de sua existência, do impacto potencial em relação à solução adotada, cumprindo-lhe elaborar ferramentas de medição e acompanhamento para ajustes na execução ou mesmo para reformulação da própria política pública.

Propõe-se, pois, a utilização do conceito de custo de transação como uma metapolítica pública, não como uma meta de política pública, que fique bem claro. A ideia não é tomá-lo como fator decisivo, mas como um importante critério que deverá juntar-se a outros, tais como os benefícios a serem alcançados, as prioridades estabelecidas nos programas de governo sufragados, os limites constitucionais e legais, os princípios regentes da Administração Pública, as particularidades dos problemas a serem enfrentados, dentre outros, sempre que se tome decisões acerca das políticas públicas, seja na fase de escolhas, de elaboração, de acompanhamento ou reformulação.

O manejo dos custos de transação deve entrar na familiaridade do gestor público. A Administração, os órgãos estatais de planejamento e estatística devem-lhe dar um tratamento diferenciado, ser capazes de identificá-los, de medi-los, de acompanhá-los, de propor alternativas institucionais que lhe levem em consideração. A política legislativa não pode ignorá-los, tampouco o Judiciário em relação ao processo de tomada de decisões.

\section{Referências}

COASE, R. H. The nature of the firm. Economica, New Series, v. 4, n. 16, p. 386-405, 1937.

COASE, R. H. The problem of social cost. The Journal of Law \& Economics, Chicago, v. 3, 1960.

COLE, D. H. The varieties of comparative institutional analysis. Wisconsin Law Review, Madison, p. 383-409, 2013.

DRIESEN, M. D.; GHOSH, S. The functions of transaction costs: rethinking transaction cost minimization in a world of friction. Arizona Law Review, Tucson, v. 47, p. 61-111, 2005.

HART, O.; HOLMSTRÖM, B. Contract Theory. Stockholm: The Royal Swedish Academy of Sciences, 2016. Disponível em: <https://www.nobelprize.org/nobel_prizes/economic-sciences/laureates/2016/advanced-economicsciences 2016.pdf>. Acesso em: 25 jun. 2017.

50 DRIESEN, M. D.; GHOSH, S. The functions of transaction costs: rethinking transaction cost minimization in a world of friction. Arizona Law Review, Tucson, v. 47, p. 61-111, 2005.

51 DRIESEN, M. D.; GHOSH, S. The functions of transaction costs: rethinking transaction cost minimization in a world of friction. Ariquna Law Review, Tucson, v. 47, p. 61-111, 2005. 
NEVES, M. A Constitucionalização simbólica. 3. ed. São Paulo: Martins Fontes, 2011.

NORTH, D. C. Institutions. Journal of Economic Perspectives, v. 5, n. 1, p. 97-112, 1991.

OSTROM, E. Beyond Markets and States: polycentric governance of complex economic systems. Prize Lecture. Workshop in Political Theory and Policy Analysis. Bloomington: Indiana University; and Tempe: Arizona State University, 2009. Disponível em: <http://www.nobelprize.org/nobel_prizes/economic-sciences/ laureates/2009/ostrom_lecture.pdf>. Acesso em: 25 jun. 2017.

SALAMA, B. M. Sete enigmas do desenvolvimento em Douglas North. Latin American and Caribbean Law and Economics Association, 2011. Disponível em: <http://abdet.com.br/site/wp-content/uploads/2014/11/ Sete-Enigmas-do-Desenvolvimento-em-Douglass-North.pdf>. Acesso em: 25 jun. 2017.

STIGLITZ, J. E. Information and the change in the paradigm in economics. prize lecture. New York: Columbia Business School, Columbia University, 2001. Disponível em: < https://edisciplinas.usp.br/pluginfile .php/17485/mod_resource/content/1/CHY\%20-\%20Stiglitz\%20-\%20Aula\%205.pdf>. Acesso em: 25 jun. 2017.

SUNSTEIN, C. R.; JOLLS, C.; THALER, R. H. A Behavioral Approach to Law and Economics. Chicago: The Law School - The University of Chicago, Program in Law and Economics Working Paper n. 55, 1998. Disponível em: <http://nrs.harvard.edu/urn-3:HUL.InstRepos:12921734>. Acesso em: 30 abr. 2017.

WILLIAMSON, O. E. Transaction Cost Economics: the natural progression. Prize Lecture. Berkeley: University of California, 2009. Disponível em: < http://www.nobelprize.org/nobel_prizes/economic-sciences/laureates/2009/ williamson_lecture.pdf>. Acesso em: 25 jun. 2017. 
Para publicar na revista Brasileira de Políticas Públicas, acesse o endereço eletrônico www.rbpp.uniceub.br

Observe as normas de publicação, para facilitar e agilizar o trabalho de edição. 